\title{
P041
}

\section{DIFFUSENESS OF ILLUMINATION SUITABLE FOR REPRODUCING THE SURFACE APPEARANCE OF OBJECTS}

\author{
Suzuki Mizushima et al. \\ DOI 10.25039/x47.2020.PO41 \\ Paper accepted for the $5^{\text {th }}$ CIE Symposium on Colour and
Visual Appearance
}

The paper was selected by the International Scientific Committee (ISC) for presentation at the 5th CIE Symposium on Colour and Visual Appearance, Hong Kong, CN, April 21-22, 2020, which, due to the corona pandemic, could not take place. The paper has not been peer-reviewed by CIE.

\section{(C) CIE 2020}

All rights reserved. Unless otherwise specified, no part of this publication may be reproduced or utilized in any form or by any means, electronic or mechanical, including photocopying and microfilm, without permission in writing from CIE Central Bureau at the address below. Any mention of organizations or products does not imply endorsement by the CIE.

This paper is made available open access for individual use. However, in all other cases all rights are reserved unless explicit permission is sought from and given by the CIE.

CIE Central Bureau

Babenbergerstrasse 9

A-1010 Vienna

Austria

Tel.: +4317143187

e-mail: ciecb@cie.co.at

www.cie.co.at 
P041

\title{
DIFFUSENESS OF THE ILLUMINATION SUITABLE FOR REPRODUCING THE SURFACE APPEARANCE OF OBJECTS
}

\author{
Mizushima, S. ${ }^{1}$, Mizokami, Y. ${ }^{1}$ \\ ${ }^{1}$ Chiba University, Chiba, JAPAN \\ suzuki-mizushima1125@chiba-u.jp
}

\begin{abstract}
The appearance of an object is influenced by its material and shape, and the lighting conditions. Especially, the diffuseness of lighting involves the appearance of material and surface textures. We investigated the diffuseness of illumination, exhibiting an accurate surface appearance compared to a memorized material recognition under natural light. We also examined the lighting condition for an ideal material appearance. After pre-observing and memorizing the impression of objects under daily lighting environments, observers evaluated the appearance of the objects under different diffuseness lighting conditions. As a result, observers highly rated moderate diffuseness compared to low and high diffuseness conditions. It implies that a very low or high diffuseness, which is unfamiliar, is not suitable for reproducing the accurate or ideal surface appearance. Our results suggest that it is possible to define a diffuseness level for accurate appearance common to objects with different materials. However, materials should be considered for the diffuseness condition for an ideal appearance.
\end{abstract}

Keywords: Diffuseness of illumination, Material perception, Surface appearance

\section{Introduction}

The impression of an object changes depending on illumination and many people experience it in everyday life. For example, a product bought under the lighting of the shop may have a different appearance when it is viewed outside. In recent years, with the evolution of solid-state lamps, including light-emitting diodes (LEDs) and organic light-emitting diodes (OLEDs), the development of lighting with various types of intensity, spectral power distribution, and spatial distribution has progressed. Besides, lamps with various shapes such as plate-type and curved designs have also been developed. These lightings could have a great difference in diffuseness from conventional lightings. Then they could have a significant impact on the appearance of objects. For example, Fig. 1 shows the appearance of an object under illumination with different diffuseness. Therefore, it is necessary to consider the surface appearance of objects such as glossiness and roughness when developing and evaluating the lighting environment.

The appearance of an object also largely depends on the material and shape. Studies on texture appearance have been conducted under various material and surface conditions (Fujisaki et al., 2015; Xiao et al., 2016; Ho et al., 2008). However, regarding the colour of an object, Maloney et al. have shown that stable colour perception can be achieved without depending on the roughness and glossiness of the surface.

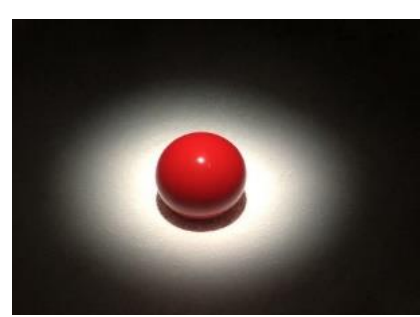

Low diffuseness

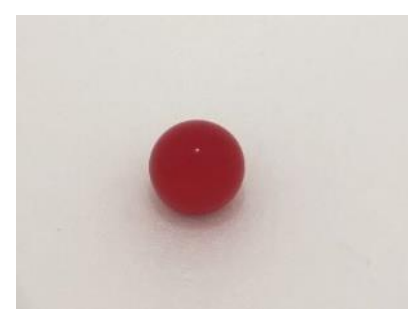

$\underline{\text { High diffuseness }}$

Figure 1 - Object appearance under lighting with different diffuseness 
The effect of surface texture has been extensively studied using images rendered using the bidirectional reflectance distribution function (BRDF) (Fleming et al., 2003; Hartung and Kersten., 2002; Langer and Bulthoff., 2001; Maloney 2002; Mingolla et al., 2001; Obein et al., 2004). It is a function that expresses the distribution of reflected light concerning the incident light. Olkkonen \& Brainard (2010) showed that the impression of matte objects was stable, but the impression of glossy objects changed when the lighting environment changed.

Lighting conditions that affect an object's appearance are related to how the light hits and how the illumination light spreads. Mizokami et al. (2019) suggested that a rough wavy surface with high frequency tends to appear less glossy and smoother as the diffuseness of the light increase. Moreover, a glossy surface appeared less glossy, and matte one appeared smoother under diffusive light. Yamazoe et al. (2019) showed that the high diffuseness of illumination increases the fidelity of the appearance of an object. However, in their experiment, the diffuseness range was limited to low to middle. Therefore, it was not yet clear how much object's appearance changes, and which diffuseness levels reproduce object's appearance more faithful and ideal. Here, we investigate the diffuseness of illumination suitable for reproducing the object's faithful or ideal surface appearance in a wider diffuseness range.

In Experiment I, we examined lighting conditions which reproduce the faithful and ideal appearance of the objects in four diffuseness levels. In Experiment II, we added a lower diffuseness condition and used a new evaluation method to clarify the lighting conditions suitable for reproducing faithful and ideal appearance.

\section{Experiment I}

\subsection{Environment}

A viewing booth was constructed by combining a lighting part and an observation part, imitating a pseudo integrating sphere, as shown in Fig. 2 (a). An observer viewed a stimulus from observation holes, as shown in Fig. 2 (b). The experiment was conducted in a dark room. The lighting part consisted of the combination of an LED lamp (Panasonic EVERLEDS LDA8DA1D; correlated colour temperature, $6500 \mathrm{~K}$; colour rendering index, Ra 73) with a duct and a Fresnel lens. The observation part was made by a white styrofoam sphere with a diameter of $60 \mathrm{~cm}$ to create a high diffuseness lighting condition.

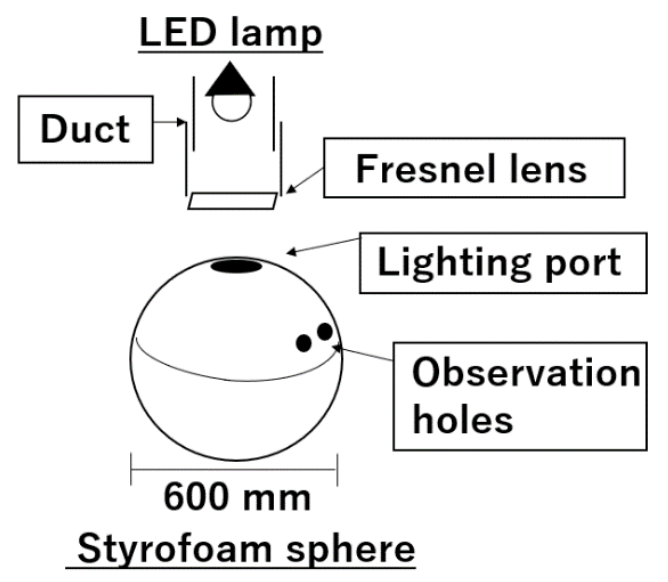

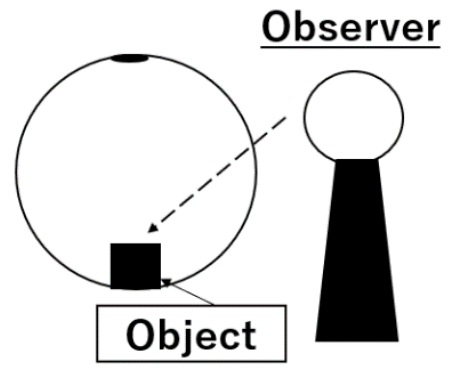

Inside of the sphere

\section{Figure 2 - (a) Viewing booth, (b) side view of the observation part}

The diffuseness of illumination was set at four levels $(0.40,0.55,0.67$, and 0.93$)$ by changing the length of the duct and the distance between the LED lamp and the lighting port, as shown in Fig. 3. We used the cubic illuminance measurement defined by Cuttle (2014) and the diffuseness value defined by Xia et al. (2016) as the measurement and evaluation method of diffuseness in our experiment. The horizontal illuminance at the position of a stimulus was unified to $300 \mathrm{Ix}$. 


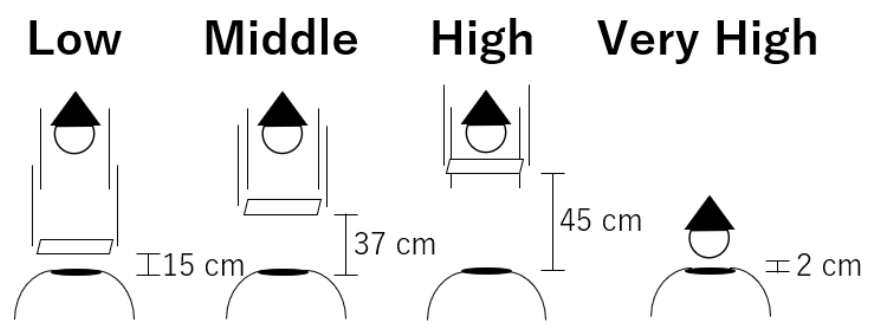

\begin{tabular}{l|llll} 
Diffuseness & $\underline{0.40}$ & $\underline{0.55}$ & $\underline{0.67}$ & $\underline{0.93}$
\end{tabular}

Figure 3 - Four-levels of diffuseness conditions

\subsection{Stimulus}

Experimental stimuli are polyresin (diameter $3.4 \mathrm{~cm}, 83 \mathrm{~g}$, sphere, red), fur charm (diameter 7 $\mathrm{cm}, 8 \mathrm{~g}$, sphere, grey), wood $(4.5 \mathrm{~cm}, 43 \mathrm{~g}$, cube, brown), and stainless steel $(2.6 \mathrm{~cm}, 35 \mathrm{~g}$, cube, silver). Figure 4 shows the experimental stimulus under each illumination.

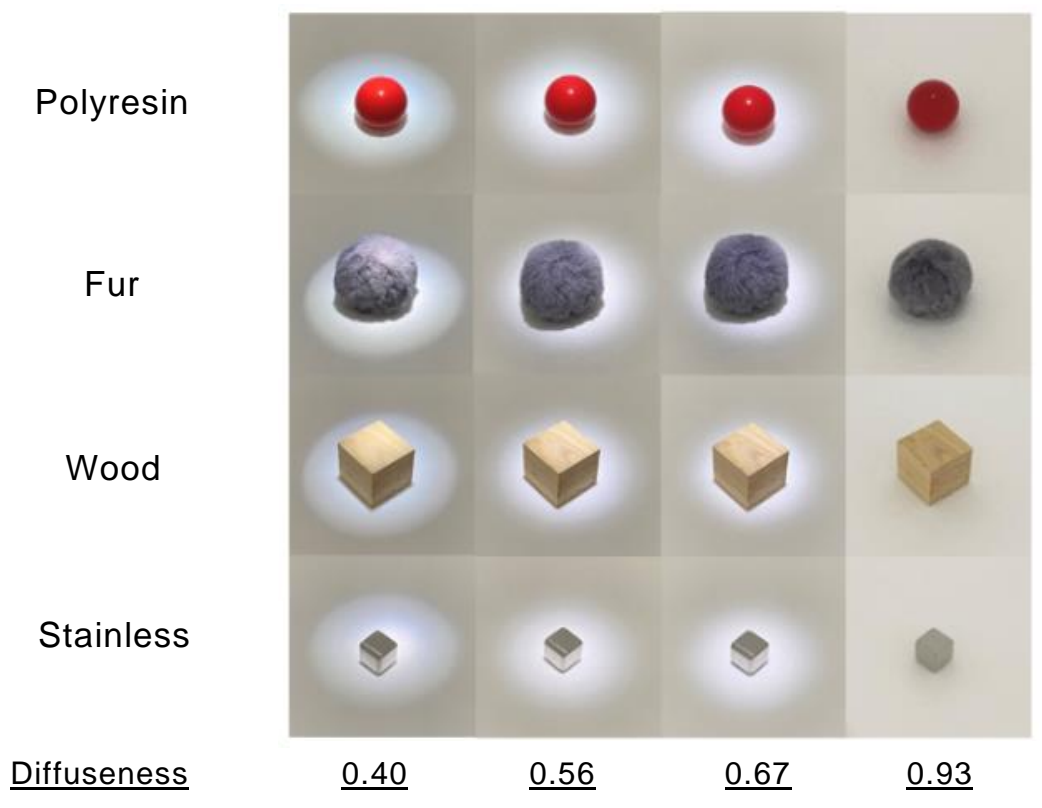

Figure 4 - Experimental stimulus of Experiment l. Polyresin, fur, wood, and stainless steel in order from the top. Diffuseness level 0.40 (Low), 0.56 (Middle), 0.67 (High), and 0.93 (Very High) in order from the left.

\subsection{Evaluation methods}

The semantic differential scale (SD) method (3-0-3) with ten items was used to evaluate the impression of the object: "Weight", "Hardness", "Naturalness", "Roughness", "Value", "Glossiness", "Preference", "Visibility", "Brightness", and "Transparency". In addition to the impression evaluation of the SD method, observers selected the best illumination condition to faithfully produce the object's appearance in their memory (fidelity illumination) and the best illumination condition to ideally produce the feature of the object's surface (ideal illumination).

\subsection{Procedure}

As a pre-observation condition, observers memorized the appearance of the stimuli by touching and viewing them at a window and in an office room for 5 minutes each for a total of 10 minutes in two separate days. The illuminance at the window ranged from $432 \mathrm{~lx}$ to $5340 \mathrm{Ix}$. The room lighting was white fluorescent lamps (National FHF32EX-NH; CCT 5000K; Ra 84), and the 
illuminance ranged from $112 \mathrm{Ix}$ to $497 \mathrm{Ix}$. As a pre-evaluation, the observers evaluated the impression of the stimuli after viewing them in the office room.

In the main evaluation, the observers viewed the stimulus in four different lighting conditions in the viewing booth, and the impression evaluation was performed. The observation time of the stimulus was unlimited, and the lighting conditions' order was randomized. After viewing the stimuli, the observer selected the fidelity illumination and the ideal illumination. The preevaluation and the main evaluation were combined into one session, and five sessions were conducted.

\subsection{Observer}

Three male and two female observers in their twenties participated.

\subsection{Result and Discussion}

The profile of the impression evaluation of observer A is shown in Fig. 5 (a). The averages of the selection rate of the fidelity illumination and ideal illumination of all observers are shown in Fig. 5 (b) and (c). Condition of very high diffuseness tends to change the impression of polyresin and stainless objects. In the faithful selection, polyresin has a high rating for low diffuseness, whereas other stimuli have a high rating for middle diffuseness. The ideal choice showed no consistent tendency overall.

For fidelity illumination, the selection ratio of "Middle (0.55)" was the highest. This diffuseness condition is the closest to the average diffuseness $(0.51)$ of the pre-observation condition. For ideal illumination, polyresin and stainless stimuli had a high selection rate for low diffuseness. Fur and wood had a high selection rate for high diffuseness.

The evaluation results of the SD method at the pre-evaluation show the characteristics of the stimuli memorized in the daily environment. Polyresin and stainless stimuli have a high score in the "glossiness" item.

The range of diffuseness during observation did not cover low diffuseness. We needed a more extensive diffuseness range of illumination to investigate a comprehensive relationship between the appearance of objects and the illumination. Therefore, we conducted Experiment II. 
$\begin{array}{llll}\text { Polyresin } & \text { Fur } & \text { Wood } & \text { Stainless }\end{array}$

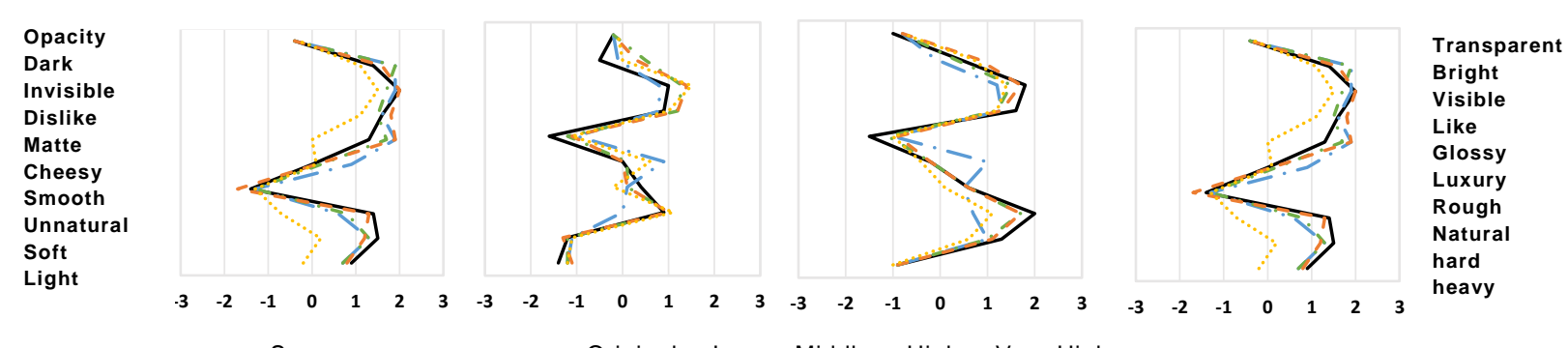

Score $\quad$-Original — Low - Middle - High - Very High

(a)

$* * \mathrm{P}<.05$

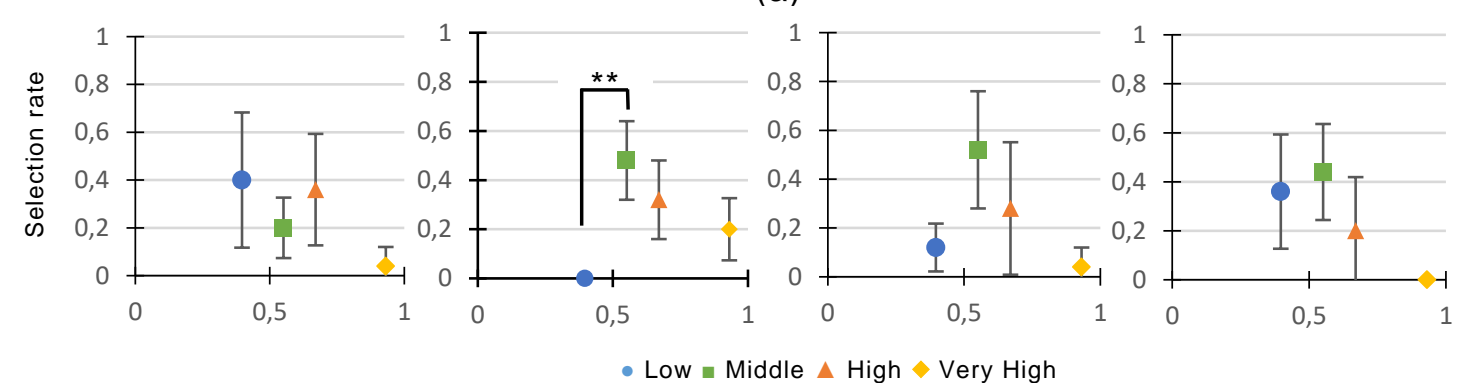

(b)

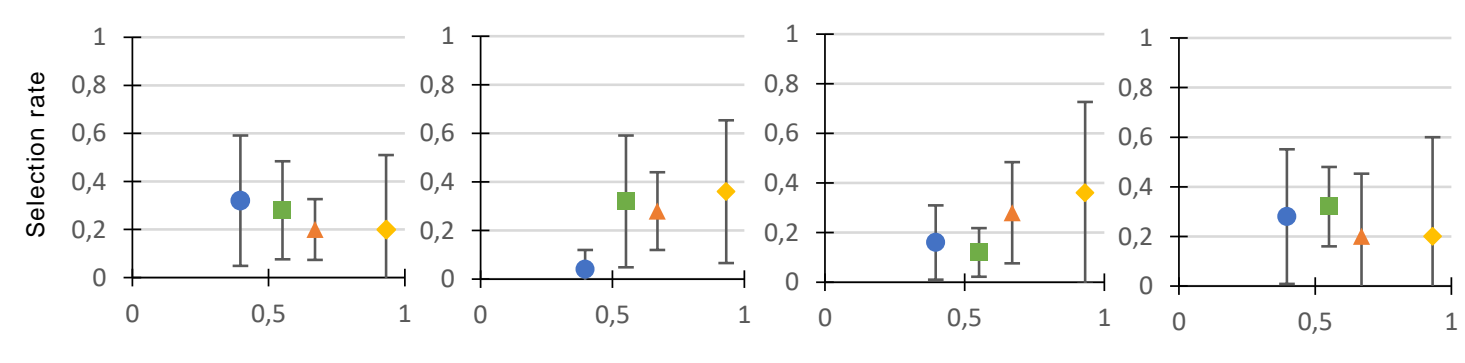

Diffuseness $\quad$ Low $=$ Middle $\Delta$ High $\diamond$ Very High

(c)

Figure 5 - Results of Experiment I. (a) The profile of the impression evaluation result of observer A. (b) the average selection rate of all observers for the fidelity illumination. (c) the average selection rate of all observers for the ideal illumination.

\section{Experiment II}

\subsection{Environment}

The viewing booth was the same as in Experiment I, except that we added a low diffuseness condition by covering the inside of the upper half of the styrofoam sphere with a black cloth to extend the diffuseness range. Therefore, we tested five diffuseness conditions $(0.26,0.40,0.55,0.67$, and 0.93$)$ in Experiment II as shown in Fig. 6. 


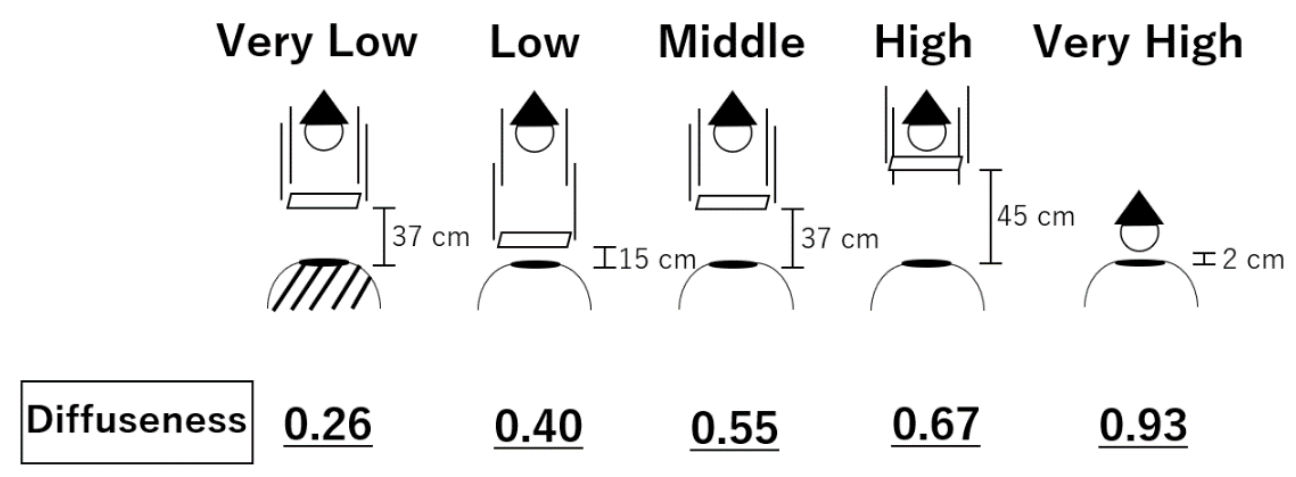

Figure 6 - Five-level diffuseness conditions

\subsection{Stimulus}

The same objects as in Experiment I was used. Figure 7 shows the state of the stimulus under each illumination.

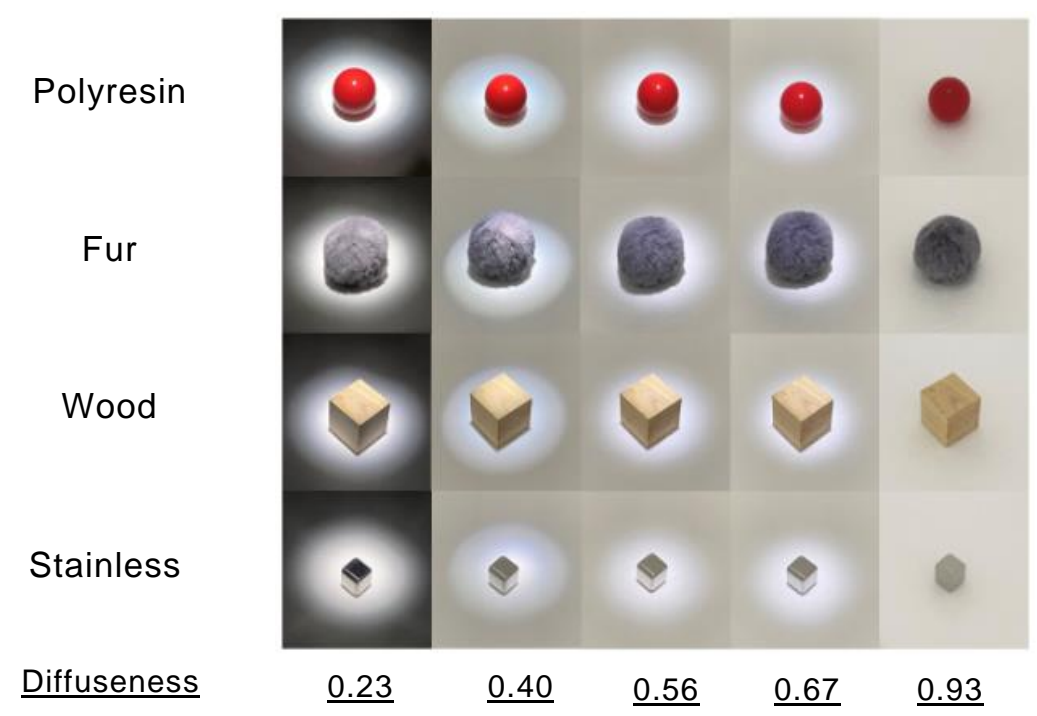

Figure 7 - Experimental stimulus of Experiment II. From top to bottom: Polyresin, fur, wood, and stainless steel. From left to right: Diffuseness level 0.23 (Very Low), 0.40 (Low), 0.56 (Middle), 0.67 (High), and 0.93 (Very High).

\subsection{Evaluation methods}

Under each diffuseness condition, observers evaluated using ten steps from 1 to 10 for the two items regarding the fidelity to the stimulus appearance memorized in the daily environment and how much the stimulus appearance was ideally represented (appearance evaluation).

\subsection{Procedure}

The pre-observation was performed in the same process as Experiment I. The illuminance at the window ranged from $51 \mathrm{~lx}$ to $4650 \mathrm{Ix}$. The illuminance inside the room ranged from $150 \mathrm{Ix}$ to $454 \mathrm{Ix}$. After viewing the stimulus for 10 minutes in the same environment as in the preobservation, the observer viewed the stimulus in the viewing booth under five lighting conditions. Then, the observer performed appearance evaluation. The observation time was unlimited, and the order of the illumination conditions was randomized. The order of presenting stimuli was also randomized, and all four types of stimuli were evaluated in one session. Five sessions were conducted. 


\subsection{Observer}

Observers were two males who participated in Experiment $I$ and two females who did not participate in Experiment I.

\subsection{Result and Discussion}

Figure 8 (a) and (b) show the average score of the fidelity and ideal lighting conditions for all observers and all stimuli. Low lighting conditions obtained a high score in the ideal evaluation for stainless, and medium score for other evaluations. Besides, Middle and High diffuseness lighting conditions had a high score in the fidelity evaluation and the ideal evaluation.

$\begin{array}{llll}\text { Polyresin } & \text { Fur } & \text { Wood } & \text { Stainless }\end{array}$

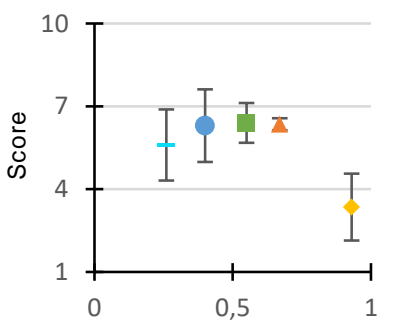

Diffusivity

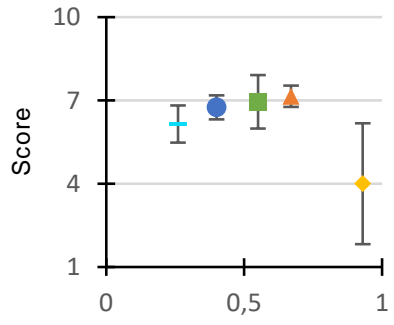

Diffusivity

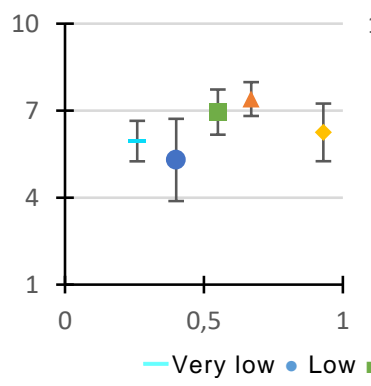

- Very low - Low

(a)
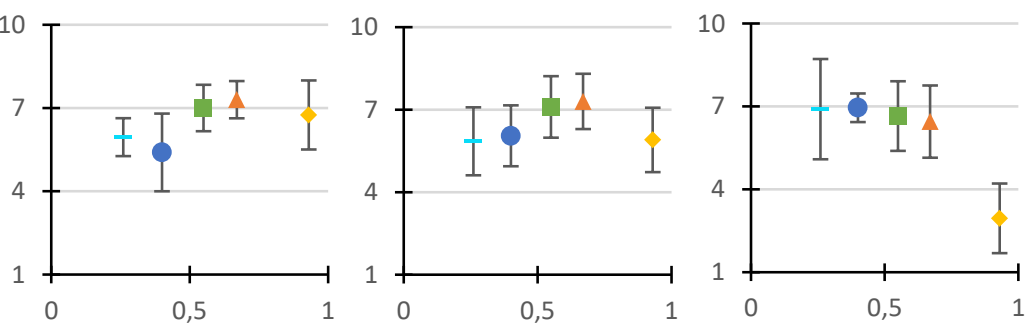

- Very low $\bullet$ Low $\approx$ Middle $\Delta$ High $\diamond$ Very High

(b)

Figure 8 - Results of Experiment II. (a) the average score of the fidelity illumination for all observers. (b) the average score of the ideal illumination for all observers.

\section{Discussion}

The results of appearance evaluations are different in Experiment I and II, probably because the evaluation method was different. In Experiment I, selecting the most faithful and ideal lighting one by one caused a large variation in evaluation. On the other hand, it was able to obtain quantitative evaluations for all stimuli in Experiment II. It was found that the scores of middle diffusenesses that were not selected in Experiment I were also high in all stimuli. There is variability in evaluation at very low and high diffuseness. It is considered that the results of Experiment II, including a large amount of data, are highly reliable.

In the experiment by Yamazoe et al. (2019), the diffuseness range was from 0.18 to 0.43 , and the fidelity increased as the diffuseness increased. In the present experiments, Very Low $(0.23)$ and Low (0.40) were within that range, and similar results to Yamazoe et al. were obtained for all objects except for fur.

The differences in evaluation between stimuli in Experiment II are similar for polyresin and stainless (P-S), and for fur and wood (F-W) in general, as shown in the average data in Table 1 and 2. In the impression evaluation in Experiment I, polyresin and stainless (P-S), and fur and wood (F-W) also had similar evaluations in average data in terms of glossiness and roughness item, as shown in Tables 3 and 4 . These results suggest that objects with similar property (e.g. 
both glossy polyresin and stainless) give shimilar impression. It can be considered that the surface appearance was influenced by the surface properties of the object.

Table 1 - Difference in appearance evaluation between stimuli (Fidelity) in Experiment II P: Polyresin, F: Fur, W: Wood, S: Stainless

\begin{tabular}{|c|r|r|r|r|r|r|}
\hline & Very Low & \multicolumn{1}{c|}{ Low } & \multicolumn{1}{c|}{ Middle } & \multicolumn{1}{c|}{ High } & \multicolumn{1}{c|}{ Very High } & \multicolumn{1}{c|}{ Average } \\
\hline P-F & 0.35 & 1.00 & 0.55 & 1.05 & 2.90 & 1.17 \\
\hline P-W & 0.35 & 0.25 & 0.60 & 0.80 & 2.65 & 0.93 \\
\hline P-S & 0.70 & 0.10 & 0.05 & 0.85 & 0.65 & 0.47 \\
\hline F-W & 0.00 & 0.75 & 0.05 & 0.25 & 0.25 & 0.26 \\
\hline F-S & 1.05 & 0.90 & 0.60 & 1.90 & 3.55 & 1.60 \\
\hline W-S & 1.05 & 0.15 & 0.65 & 1.65 & 3.30 & 1.36 \\
\hline
\end{tabular}

Table 2 - Difference in appearance evaluation between stimuli (Ideal) in Experiment II

\begin{tabular}{|c|r|r|r|r|r|r|}
\hline & Very Low & \multicolumn{1}{|c|}{ Low } & \multicolumn{1}{c|}{ Middle } & \multicolumn{1}{c|}{ High } & \multicolumn{1}{c|}{ Very High } & \multicolumn{1}{c|}{ Average } \\
\hline P-F & 0.20 & 1.35 & 0.05 & 0.15 & 2.75 & 0.90 \\
\hline P-W & 0.30 & 0.70 & 0.15 & 0.15 & 1.90 & 0.64 \\
\hline P-S & 0.75 & 0.20 & 0.30 & 0.70 & 1.05 & 0.60 \\
\hline F-W & 0.10 & 0.65 & 0.10 & 0.00 & 0.85 & 0.34 \\
\hline F-S & 0.95 & 1.55 & 0.35 & 0.85 & 3.80 & 1.50 \\
\hline W-S & 1.05 & 0.90 & 0.45 & 0.85 & 2.95 & 1.24 \\
\hline
\end{tabular}

Table 3 - Difference in impression evaluation between stimuli (Glossiness) in Experiment I

\begin{tabular}{|c|r|r|r|r|r|r|}
\hline & \multicolumn{1}{|c|}{ Original } & \multicolumn{1}{c|}{ Low } & \multicolumn{1}{c|}{ Middle } & \multicolumn{1}{c|}{ High } & \multicolumn{1}{c|}{ Very High } & \multicolumn{1}{c|}{ Average } \\
\hline P-F & 2.84 & 2.68 & 2.32 & 2.30 & 1.90 & 2.41 \\
\hline P-W & 2.40 & 2.66 & 2.74 & 2.28 & 1.44 & 2.30 \\
\hline P-S & 0.80 & 0.28 & 0.22 & 0.10 & 0.46 & 0.37 \\
\hline F-W & 0.44 & 0.02 & 0.42 & 0.02 & 0.46 & 0.27 \\
\hline F-S & 3.64 & 2.96 & 2.54 & 2.40 & 1.44 & 2.60 \\
\hline W-S & 3.20 & 2.94 & 2.96 & 2.38 & 0.98 & 2.49 \\
\hline
\end{tabular}

Table 4 - Difference in impression evaluation between stimuli (Roughness) in Experiment I

\begin{tabular}{|c|r|r|r|r|r|r|}
\hline & \multicolumn{1}{|c|}{ Original } & \multicolumn{1}{c|}{ Low } & \multicolumn{1}{c|}{ Middle } & \multicolumn{1}{c|}{ High } & \multicolumn{1}{c|}{ Very High } & \multicolumn{1}{c|}{ Average } \\
\hline P-F & 2.60 & 2.40 & 2.40 & 2.42 & 2.36 & 2.44 \\
\hline P-W & 1.54 & 1.86 & 1.42 & 1.66 & 0.90 & 1.48 \\
\hline P-S & 0.44 & 0.14 & 0.08 & 0.00 & 0.06 & 0.14 \\
\hline F-W & 1.06 & 0.54 & 0.98 & 0.76 & 1.46 & 0.96 \\
\hline F-S & 3.04 & 2.26 & 2.48 & 2.42 & 2.30 & 2.50 \\
\hline W-S & 1.98 & 1.72 & 1.50 & 1.66 & 0.84 & 1.54 \\
\hline
\end{tabular}


The reason why the middle diffuseness was highly evaluated would be because it was within the diffuseness range at pre-observation, where observers memorized the appearance of objects. The other possibility would be related to the naturalness of lighting conditions. Fleming et al. (2003) revealed that the reflection properties of objects in familiar environments are easier to distinguish than in unfamiliar environments. It can be considered that the moderate diffuseness was preferred because it is commonly seen in our ordinary life. Our results suggest that unfamiliar lighting conditions (very low and very high diffuseness) did not correctly represent the appearance of objects.

\section{Conclusion}

In this study, the observers viewed objects with different materials, shapes, and textures. They evaluated the impression of those objects, as well as the fidelity and the ideality of objects' appearance under different diffuseness levels of illumination. The results showed that illumination with middle diffuseness reproduced the appearance of objects faithful and ideally, and extremely low and high diffuseness were not. It was also suggested that the diffuseness, which ideally reproduces the surface appearance, would be different in object's properties.

To summarize, we showed that the impression of surface appearance was influenced by the surface properties of the object and lighting distribution. Our results suggest that it is possible to define a diffuseness level for accurate appearance common to objects with different materials. However, the difference in the material should be considered for the diffuseness condition for an ideal appearance to realize the ideal appearance.

\section{Acknowledgments}

Supported by JSPS KAKENHI JP16K00368 and JP19H04196.

\section{References}

CUTTLE, C. 2014. Research note: A practical approach to cubic illuminance measurement. Lighting Research \& Technology, 46(1), 31-34.

FLEMING, W. R., DROR, O. R., ADELSON, H. E. 2003. Real-world illumination and the perception of surface reflectance properties. Journal of Vision, 3(5), 347-368.

FLEMING, R. W., DROR, R. O., ADELSON, E. H. 2003. Surface reflectance estimation under unknown natural illumination. Journal of Vision, 1(3), 43.

FUJISAKI, W., TOKITA, M., KARIYA, K. 2015. Perception of the material properties of wood based on vision, audition, and touch. Vision Research, 109, 185-200.

HARTUNG, B., KERSTEN, D. 2002. Distinguishing shiny from matte. Journal of Vision, 2(7), 551.

HO, Y. X., LANDY, M. S., MALONEY, L. T. 2008. Conjoint measurement of gloss and surface texture. Psychological science, 19(2), 196-204.

LANGER, M. S., BULTHOFF, H. H., 2001. A prior for global convexity in local shape-fromshading. Perception, 30(4), 403-410.

MALONEY, L. T., GERHARD, H. E., BOYACI, H. DOERSCHNER, K. 2011. Surface color perception and light field estimation in 3D scenes. Natural-scene perception. Journal of Vision, 1(3), 280-307.

MAMASSIAN, P., LANDY, M. S., MALONEY, L. T. 2002. Bayesian Modelling of Visual Perception. Probabilistic Models of brain: Perception and Neural Function (13-36). Cambridge, MA: MIT Press.

MIZOKAMI, Y., KIYASU, Y., YAGUCHI, H. 2019. Change in the appearance of objects according to the ratio of direct and diffusive light. CIE X046: 2019 Proceedings of the 29th CIE SESSION Washington DC, USA, DOI 10.25039/x46.2019.PP05. 
OBEIN, G., KNOBLAUCH, K., VIE 'NOT, F. 2004. Difference scaling of gloss: Nonlinearity, binocularity, and constancy. Journal of Vision, 4(9), 711-720.

OLKKONEN, M., BRAINARD, D. H. 2010. Perceived glossiness and lightness under real-world illumination. Journal of Vision, 10(9), 1-19.

PONT, S. C., TE PAS, S. F. 2006. Material-Illumination ambiguities and the perception of solid objects. Perception, 35(10), 1331-1350.

XIAO, B., BI, W., JIA, X., WEI, H., ADELSON, E. H. 2016. Can you see what you feel? Color and folding properties affect visual-tactile material discrimination of fabrics. Journal of Vision, 16(3), 34.

XIA, L., PONT, S. C., HEYNDERICKX, I. 2016. Diffuseness metric Part 1: Theory. Lighting Research \& Technology, 49(4), 411-427.

YAMAZOE, T., FUNAKI, T., KIYASU, Y., MIZOKAMI, Y. 2019. Evaluation of material appearance under different spotlight distributions compared to natural illumination. Journal of Imaging, 5(2), 31. 